Editorial

\title{
The diverse and rapidly growing HIV epidemic in the WHO Eastern Mediterranean Region: will countries succeed in their control?
}

\author{
Gabriele Riedner ${ }^{7}$
}

More than 30 years into the HIV epidemic, there is growing confidence in the world's ability to lay the foundation for an AIDS-free generation, fuelled by accumulating evidence of the therapeutic and preventive benefits of ART [1]. Do countries in the WHO Eastern Mediterranean Region (EMR) have reason to join this optimism?

In 2012, 490000 people in the EMR were living with HIV, a 69\% increase over the number living with HIV in 2002 [2]. Even as new infections continue to decline worldwide, there are disturbing signs that the Region's epidemic is worsening. Among the world's regions, HIV incidence is increasing fastest in the EMR, with the annual number of new infections rising from 41000 in 2002 to 63000 in 2012, i.e. a $54 \%$ increase.

It is noteworthy that HIV prevalence remained low in the general population in most countries of the Region. This is likely to be attributable to prevalent cultural values, norms and practices including universal male circumcision, prohibition of sexual relations outside marriage and alcohol abstinence [3]. However, cultural norms also tend to result in stigma and discrimination against people living with HIV and populations at higher risk, thus posing major barriers to reaching them with HIV prevention, testing, care and treatment [4].

A growing number of epidemiological studies in the Region confirm the diversity of HIV epidemics in the
Region [5]. Considerable geographic variation in HIV burden is reported between but also within individual countries. Moreover, while the percentage of the general adult population living with HIV in the Region is among the lowest globally, certain populations have been disproportionately affected. These populations include men who have sex with men, people who inject drugs, prisoners and sex workers. Recent surveys report elevated HIV prevalence among people who inject drugs in the Islamic Republic of Iran, Pakistan, Afghanistan, Egypt, Libya, Tunisia and Morocco with levels reaching up to $87 \%$ in Libya $[6,7]$. HIV epidemics among men who have sex with men are reported from Morocco, Tunisia, Egypt, Sudan, Yemen and Pakistan [9] and among female sex workers in Morocco, Sudan and Djibouti [9]. Data on HIV among populations at higher risk are still lacking in many countries and HIV epidemics among these populations may thus be hidden and growing silently.

How are countries in the Region responding to these challenges?

Some have gained deeper insight into the dynamics of the HIV epidemics and have made commendable strides to focus their prevention efforts on populations most in need, to increase coverage of HIV testing, to enrol pregnant women in services to prevent mother-to-child transmission and to reach an increasing number of people living with HIV (PLHIV) with life-saving antiretroviral therapy. Success can be felt in 2012 when the number of PLHIV receiving antiretroviral therapy in the Region is estimated to have increased from 23500 to 33000. Despite this progress, region-wide coverage of antiretroviral therapy, as well as prevention of mother-to-child transmission services, remains the lowest in the world [10].

National AIDS programmes in most countries continue to face major challenges in terms of political commitment of national leaders, financial resources, health and community system weaknesses and cultural acceptability of public health approaches to HIV prevention and control [11]. The average per capita spending on HIV prevention and control in the Region was 5 cents per capita: the lowest globally [12].

Turning the tide of the HIV epidemic in the Region will require leadership, determination and cooperation of senior national leaders, health ministries, parliamentarians, health professional groups, the private sector, religious leaders and civil society, especially PLHIV. Bold action is needed to de-stigmatize HIV and to eliminate discrimination. HIV testing and treatment must be brought closer to people in need. The use of new simplified high-quality laboratory technologies and the adoption of affordable "one pill per day" antiretroviral therapy regimens will facilitate decentralization of services to community settings while assuring quality [13]. 


\section{References}

1. Fauci AS. AIDS: let science inform policy. Science, 2011, 333:13.

2. Global report: UNAIDS report on the global AIDS epidemic 2013. Geneva, Joint United Nations Programme on HIV/AIDS (UNAIDS), 2013.

3. Alkaiyat A, Weiss MG. HIV in the Middle East and North Africa: priority, culture, and control. International Journal of Public Health, 2013 [Epub ahead of print].

4. Abu-Raddad L et al. Characterizing the HIV/AIDS epidemic in the Middle East and North Africa: time for strategic action. Washington DC, World Bank, 2010.

5. Bozicevic I, Riedner G, Calleja JM. HIV surveillance in MENA: recent developments and results. Sexually Transmitted Infections, 2013 [Epub ahead of print].

6. Bozicevic I, Riedner G, Calleja JM. HIV surveillance in MENA: recent developments and results. Sexually Transmitted Infections, 2013 Feb 23 [Epub ahead of print].

7. Mirzoyan L et al. New evidence on the HIV epidemic in Libya: why countries must implement prevention programs among people who inject drugs. Journal of Acquired Immune Deficiency Syndromes, 2013 62(5):577-583
8. Mumtaz G et al. Are HIV epidemics among men who have sex with men emerging in the Middle East and North Africa? A systematic review and data synthesis. PLoS Medicine, 2010, 8:e1000444.

9. WHO. HIV Surveillance in the WHO Eastern Mediterranean Region. Regional Update, 2013, 2012 (accepted for publication).

10. WHO, UNICEF, UNAIDS. Global update on HIV treatment: results, impact and opportunities. Geneva, World Health Organization, 2013.

11. Accelerating HIV reatment in the WHO Eastern Mediterranean/ UNAIDS Middle East and North Africa Region. Cairo, WHO/ UNAIDS, 2013.

12. Ávila $\mathrm{C}$ et al. Determinants of government HIV/AIDS financing: a 10-year trend analysis from 125 low- and middle-income countries. BMC Public Health, 2013, 13:673

13. Consolidated guidelines on the use of antiretroviral drugs for treating and prevention HIV infection. Recommendations for a public health approach. Geneva, World Health Organization, 2013. 